\title{
Libros infantiles con temática relacionada con la Educación Física y el Deporte en Brasil: estudio de sus características
}

\author{
Rafael Guimarães Botelho \\ Instituto Federal de Educación, Ciência y Tecnología de Río de Janeiro (IFRJ) - Brasil \\ Facultad de Educación de la Universidad de San Pablo (USP) - Brasil
}

ARTÍCULO / ARTICLE

\begin{abstract}
Resumen
El objetivo de este artículo ha sido analizar 90 libros infantiles con temática relacionada con la Educación Física y el Deporte publicados en Brasil. Han sido establecidas cinco variables de estudio: géneros literarios, soportes, paginación, vinculación a una colección sobre deportes y versión de la publicación. Para llevar a cabo la investigación, se ha utilizado la técnica del análisis documental. Resultados: a) la narrativa presenta la mayor incidencia en las obras infantiles (con un 93,33\%); b) el soporte papel es el predominante en los libros infantiles (con un $90 \%$ ); c) en las obras publicadas en Brasil prevalecen los libros no paginados (con un $73,33 \%$ ); d) poco más de la mitad (con un $51,11 \%$ ) de los libros infantiles está vinculado a una colección sobre deportes; e) las obras infantiles originales son las predominantes (con un 96,67\%).
\end{abstract}

Palabras clave

Literatura infantil ; Educación Física ; Análisis documental ; Brasil

\section{Children's books dealing with Physical Education and Sport in Brazil: a study of their characteristics}

\begin{abstract}
The aim of this paper was to analyze 90 children's books dealing with Physical Education and Sport published in Brazil. Five study variables were established: literary genres, mediums, pagination, status as part of a collection related to sports, and the version of the publication. To develop the investigation, the technique of documentary analysis was utilized. Results: a) the literary genre "narrative" presents the biggest incidence in the children's books (with 93,33\%); b) the medium "paper" is predominant in the children's books (with 90\%); c) books not paginated are more frequent among the ones published in Brazil (with $73,33 \%)$; d) a little more than half of the children's books $(51,11 \%)$ is part of a collection related to sports; e) there are a predominance of original children's books (with 96,67\%).
\end{abstract}

Keywords

Children's literature ; Physical Education ; Documentary analysis ; Brazil

\section{Introducción}

Diferentemente del ámbito internacional, en el que ya se constata la existencia de trabajos que investigan los libros de literatura infantil y su relación con el Deporte (Botelho \& Oliveira 2011; Fingon, 2011; Weiller \& Higgs, 1989), en Brasil aún son embrionarios los textos académicos en el área de Educación Física que analizan los libros infantiles.

Como consecuencia, todavía queda mucho camino por recurrir cuando el asunto es conocer las características de las obras infantiles con temática relacionada con el Deporte. 
Por ejemplo, interrogantes como ¿cuáles son las características de los libros infantiles con temática relacionada con la Educación Física y el Deporte publicados en Brasil?, son preguntas aún sin respuestas.

Con el fin de dar respuesta a la pregunta planteada, el objetivo de este artículo es analizar 90 obras infantiles con temática relacionada con la Educación Física y el Deporte publicadas en Brasil.

\section{Materiales y método}

\subsection{El análisis documental}

Para llevar a cabo el estudio de los 90 libros infantiles se ha utilizado la técnica del análisis documental.

Para Jacques Chaumier (1993) el análisis documental es "una operación o un conjunto de operaciones encaminadas a representar el contenido de un documento bajo una forma diferente de su forma original, con la finalidad de facilitar su consulta o su localización en una fase posterior" (p. 17).

En realidad, "el análisis documental permite pasar de un documento primario (bruto) a un documento secundario (representación del primero). Esto es lo que son, por ejemplo, los resúmenes o extractos (condensación del documento según ciertas reglas)" (Bardin, 2002, p. 35).

Por lo tanto, este tipo de análisis permite el control documental y la recuperación de información (López Yepes, 2004).

\subsection{Descripción y delimitaciones del objeto de estudio}

A continuación, la Figura 1 describe las delimitaciones establecidas para la organización y selección de las obras de literatura infantil.

\section{Descripción del objeto de estudio}

Objeto de estudio. Noventa (90) libros infantiles publicados originalmente en lengua portuguesa o traducidos a este idioma. Las referencias de estos libros se encuentran en la tesis doctoral Educación Física y literatura infantil: posibilidades de utilización en el ámbito escolar (Botelho, 2010).

\section{Delimitaciones del objeto de estudio}

Temática. Relacionada con la Educación Física y el Deporte.

Lugar de publicación. Editoriales con sede en Brasil o empresas y sociedades limitadas de traducción también con sede en Brasil.

Temporal. En este trabajo se investigaron los libros infantiles publicados en un período de, aproximadamente, 22 años (desde 1988 hasta 2009).

Número de páginas / ilustraciones. El número de páginas y la relación entre la cantidad de texto y de ilustraciones de las obras infantiles son aspectos importantes a tener en cuenta. Por esta razón, se han excluido del corpus de análisis los libros con más de 65 páginas o libros infantiles que no presentan ilustraciones.

Figura 1 - Descripción y delimitaciones del objeto de estudio 


\subsection{Descripción de las variables}

Para Hernández Sampieri, Fernández Collado y Baptista Lucio (2007) variable es la "propiedad que puede variar o adquirir distintos valores y cuya variación es susceptible de medirse u observarse" (p. 76). Por tanto, a continuación, se presentan las variables y sus respectivos códigos establecidos en la investigación.

\begin{tabular}{|c|c|}
\hline Variables & Código: \\
\hline \multirow{2}{*}{ Géneros literarios } & 1. Narrativa \\
\hline & 2. Poesía \\
\hline \multirow{7}{*}{ Soportes } & 1. Papel \\
\hline & 2. Disco \\
\hline & 3. Internet \\
\hline & 4. Papel y disco \\
\hline & 5. Papel e Internet \\
\hline & 6. Disco e Internet \\
\hline & 7. Papel, disco e Internet \\
\hline \multirow{2}{*}{ Paginación } & 1. Paginado \\
\hline & 2. No paginado \\
\hline \multirow{2}{*}{ Vinculación a una colección sobre deportes } & 1. Sí \\
\hline & 2. No \\
\hline \multirow{2}{*}{ Versión de la publicación } & 1. Original \\
\hline & 2. Traducción \\
\hline
\end{tabular}

Figura 2 - Cuadro con las variables de análisis y sus respectivos códigos

\section{Resultados}

\subsection{Presentación y discusión}

Este artículo investigó 90 libros publicados en Brasil en un período de, aproximadamente, 22 años (1988-2009), aunque 84 de las obras pertenecen a la primera década del año 2000.

\subsection{Géneros literarios}

La Tabla 1 registra los valores obtenidos de la primera variable. 


\begin{tabular}{|c|c|}
\hline Géneros literarios & $\begin{array}{c}\text { Frecuencias } \\
\text { absolutas }\end{array}$ \\
\hline 1- Narrativa & 84 \\
\hline 2- Poesía & 6 \\
\hline Total & 90 \\
\hline
\end{tabular}

Tabla 1 - Frecuencia absoluta de los géneros literarios de las obras infantiles

Los resultados de esta tabla indican que: (a) la narrativa presenta la mayor incidencia, con 84 obras; (b) la poesía aparece en solamente seis libros infantiles.

La Figura 3 constata una gran desproporción entre los libros de narrativa y los de poesía.

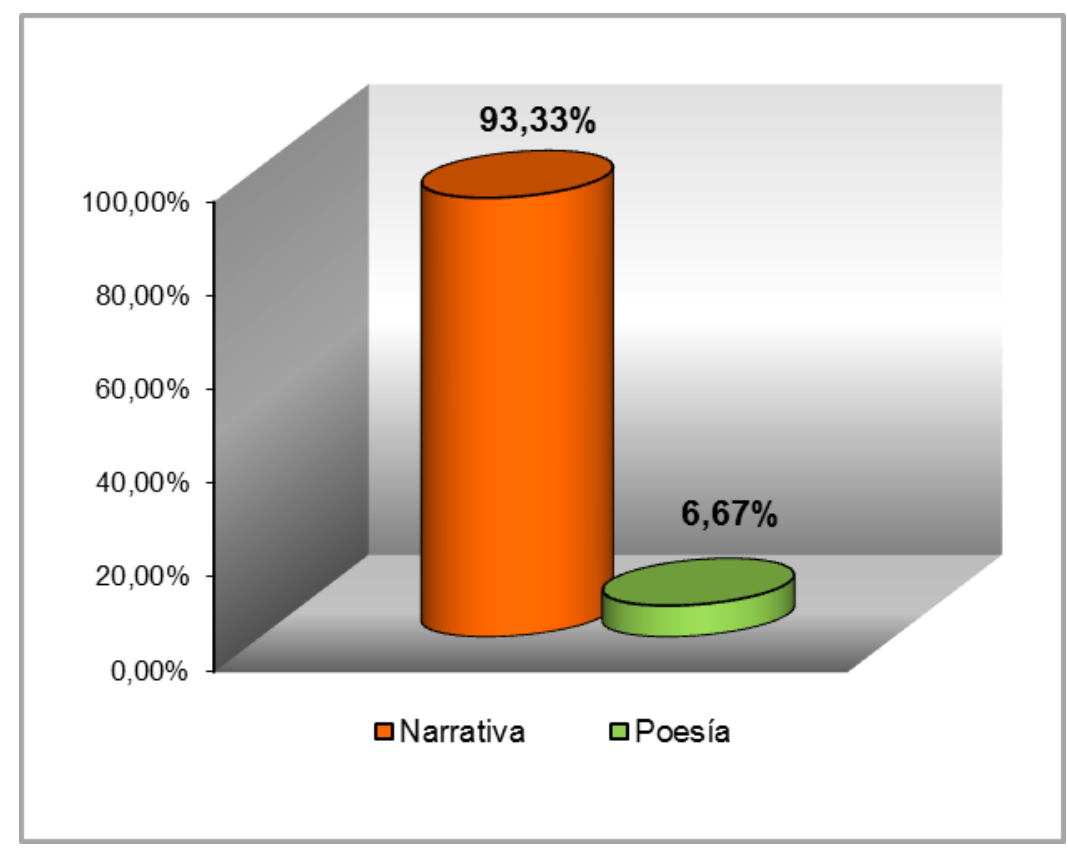

Figura 3 - Representación gráfica de la frecuencia relativa de los géneros literarios de las obras infantiles

En esta figura se verifica que la narrativa es el género predominante en los libros infantiles publicados en Brasil, con un $93,33 \%$. En cambio, la poesía está presente únicamente en $6,67 \%$ de las obras analizadas.

La constatación de que la poesía es un género poco difundido en los libros publicados en Brasil es un dato negativo, ya que, como señala Abril Villalba (2005), "los textos poéticos ... pueden servir como instrumento de apoyo a los juegos" (p. 206).

\subsection{Soportes}

Se identifican dos ítems de los siete posibles, lo que puede observarse en la Tabla 2, presentada a continuación. 


\begin{tabular}{lc}
\hline Soportes & $\begin{array}{c}\text { Frecuencias } \\
\text { absolutas }\end{array}$ \\
\hline 1- Papel & 81 \\
2- Disco & 0 \\
3- Internet & 0 \\
4- Papel y disco & 9 \\
5- Papel e Internet & 0 \\
6- Disco e Internet & 0 \\
7- Papel, disco e Internet & 0 \\
\hline Total & 90 \\
\hline
\end{tabular}

Tabla 2 - Frecuencia absoluta de los tipos de soportes de las obras infantiles

Los resultados de esta tabla señalan que: (a) el soporte papel tiene la mayor incidencia, con 81 libros; (b) el ítem papel y disco presenta nueve libros; (c) no se registra ninguna incidencia de los demás soportes.

En la Figura 4 se observa que el papel es aún el soporte predominante en la publicación de las obras infantiles.

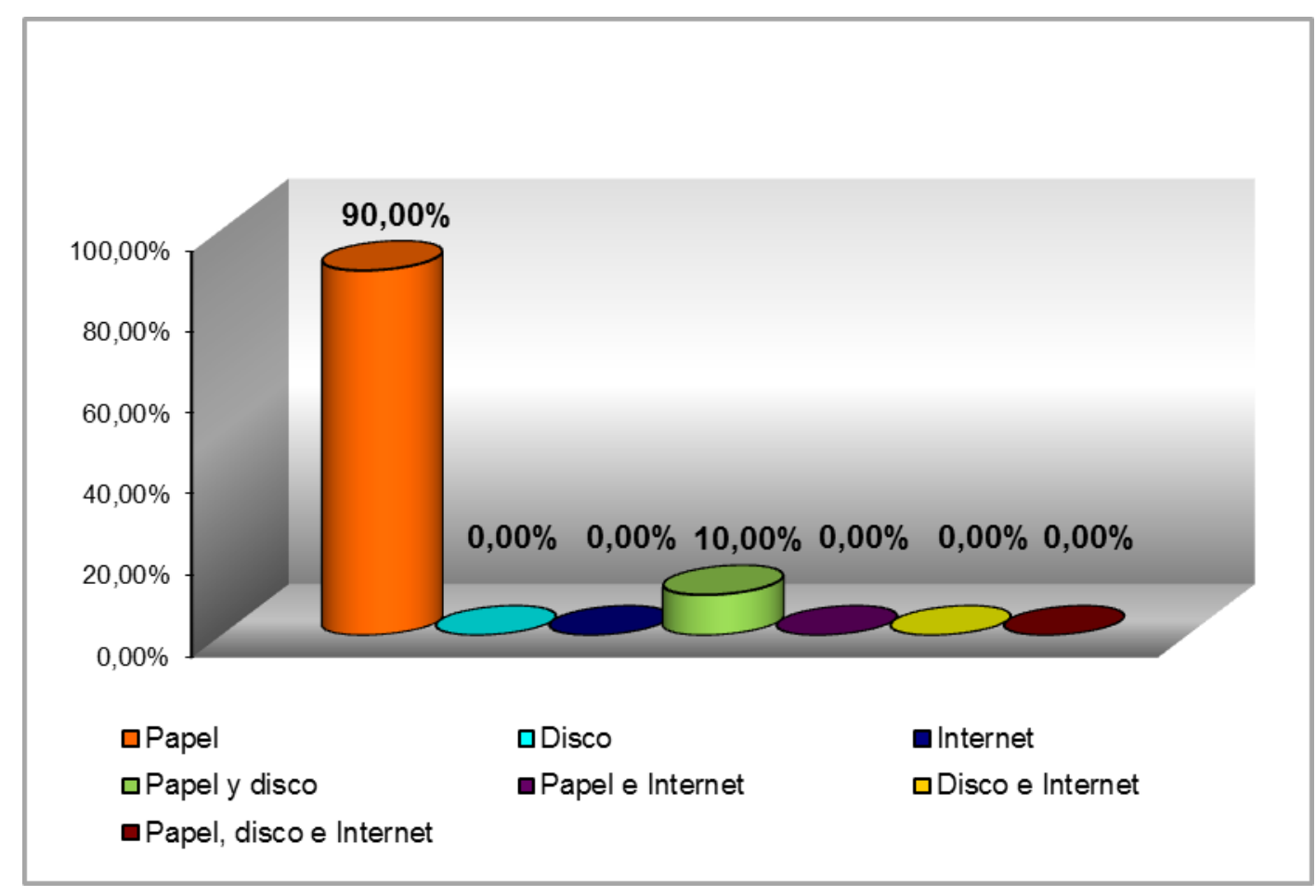

Figura 4 - Representación gráfica de la frecuencia relativa de los tipos de soportes de las obras infantiles

En esta figura se registra una asimetría entre los tipos de soporte. El papel (con un 90\%) es el soporte predominante en los libros, mientras que los libros infantiles en soporte papel y disco alcanzan un 10,00\%. 
A partir de estos datos, se puede desprender que la relación entre los libros con temática relacionada con la Educación Física y el Deporte y las Tecnologías de la Información y de la Comunicación (TIC) es aún incipiente y, por eso, necesita más atención, creatividad e inversión por parte de los autores y de las editoriales en Brasil.

\subsection{Paginación}

Con relación a la variable paginación, la Tabla 3 presenta los siguientes resultados.

\begin{tabular}{|c|c|}
\hline Paginación & $\begin{array}{c}\text { Frecuencias } \\
\text { absolutas }\end{array}$ \\
\hline 1- Paginado & 24 \\
\hline 2- No paginado & 66 \\
\hline Total & 90 \\
\hline
\end{tabular}

Tabla 3 - Frecuencia absoluta de la paginación de las obras infantiles

Esta tabla revela los siguientes valores: (a) se registran 24 obras infantiles paginadas; (b) se identifican 66 libros infantiles no paginados.

En la Figura 5 se comprueba una gran asimetría entre los libros infantiles paginados y no paginados publicados en Brasil.

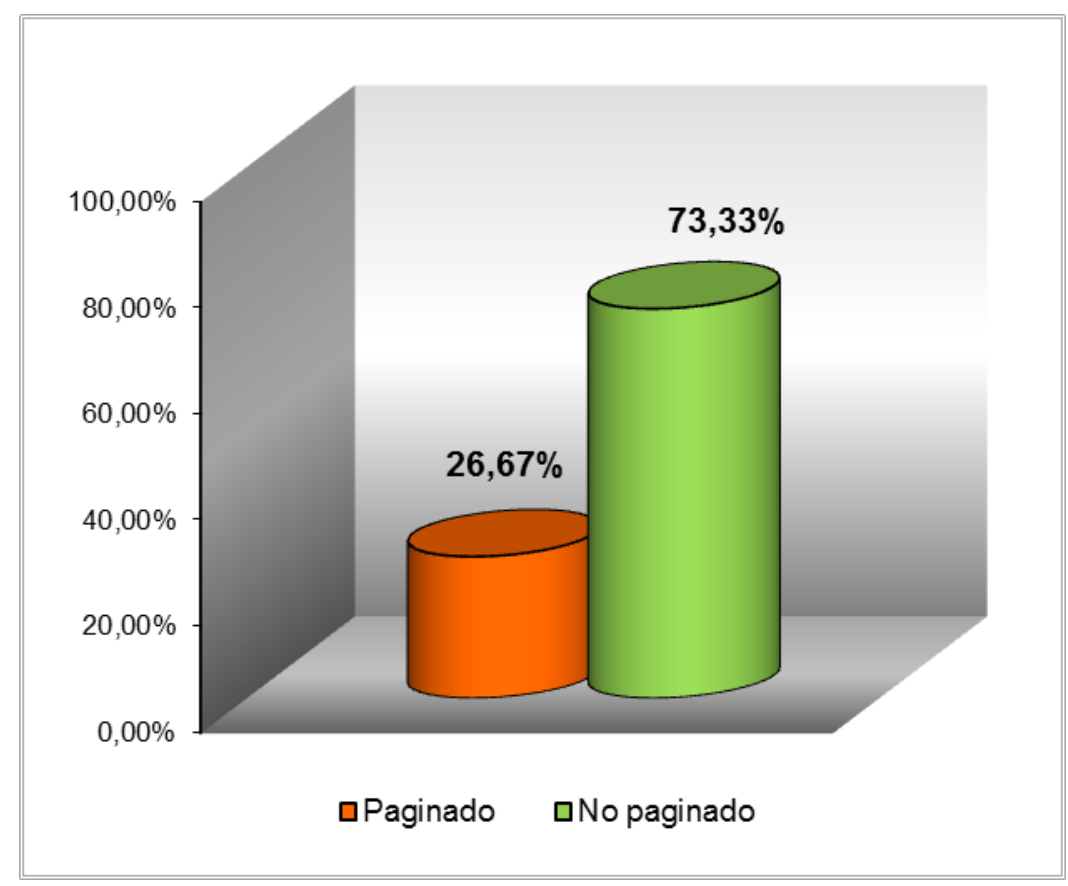

Figura 5 - Representación gráfica de la frecuencia relativa de la paginación de las obras infantiles

En el caso de las obras publicadas en Brasil prevalecen los libros no paginados, con un 73,33\%. Muy por debajo de eso, con un $26,67 \%$, están los libros paginados. 
La paginación es parte de la organización formal de una obra. Es más visible en los libros académicos y científicos, en los que hay la necesidad de identificar de manera precisa las fuentes de información.

Sin embargo, en los libros infantiles la paginación puede ser un elemento importante para que el niño haga una lectura lineal del texto. Los libros paginados permiten que el niño no se pierda en el transcurso de la narrativa. Además, es un indicador esencial para que el profesorado sepa elegir las obras adecuadas de acuerdo con el nivel de lectura y la edad del niño y, también, para el tiempo que dispone el maestro o profesor en una sesión de Educación Física.

\subsection{Vinculación a una colección sobre deportes}

Respecto a esta variable, la Tabla 4 presenta los valores identificados en las obras infantiles.

\begin{tabular}{lc}
\hline \multicolumn{1}{c}{$\begin{array}{c}\text { Vinculación a una colección sobre } \\
\text { deportes }\end{array}$} & $\begin{array}{c}\text { Frecuencias } \\
\text { absolutas }\end{array}$ \\
\hline 1- Sí & 46 \\
2 - No & 44 \\
\hline Total & 90 \\
\hline
\end{tabular}

Tabla 4 - Frecuencia absoluta de la vinculación a una colección sobre deportes de las obras infantiles

Los valores presentes en esta tabla indican que: (a) se identifican 46 obras infantiles vinculadas a una colección sobre deportes; (b) se registran 44 libros infantiles que no pertenecen a una colección sobre deportes.

Respecto a esta variable, la Figura 6 presenta un equilibrio entre sus ítems.

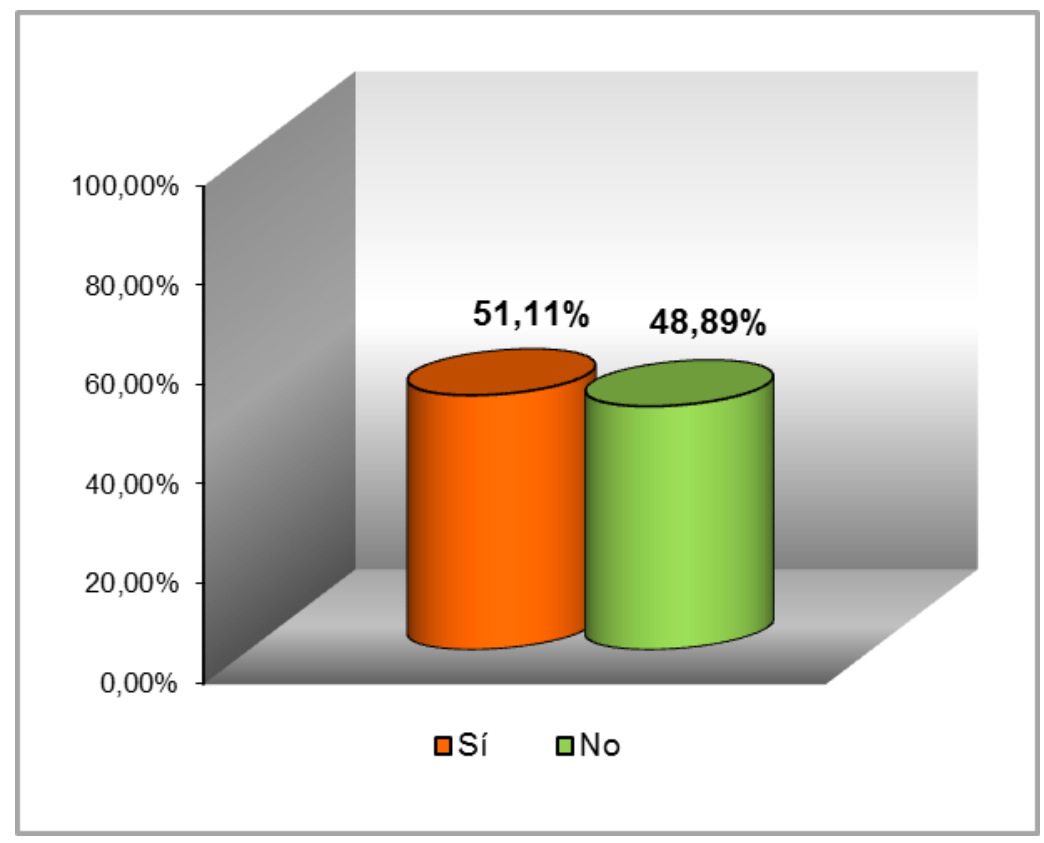

Figura 6 - Representación gráfica de la frecuencia relativa de la vinculación a una colección sobre deportes de las obras infantiles 
Esta figura demuestra que poco más de la mitad (un 51,11\%) de los libros infantiles está vinculado a una colección sobre deportes.

Sin lugar a dudas, es importante para el área de Educación Física que los libros infantiles estén vinculados a una colección con temática relacionada con el Deporte, porque facilita el desarrollo de los diferentes enfoques, pudiendo o no utilizar los mismos personajes en la colección infantil. Además, es más fácil identificar, adquirir e incluir los libros infantiles de una colección en diferentes sesiones de Educación Física que localizar los libros infantiles publicados separadamente.

\subsection{Versión de la publicación}

La Tabla 5 revela una gran diferencia entre las obras infantiles originales y las traducidas.

\begin{tabular}{lc}
\hline \multicolumn{1}{c}{ Versión de la publicación } & $\begin{array}{c}\text { Frecuencias } \\
\text { absolutas }\end{array}$ \\
\hline 1- Original & 87 \\
2- Traducción & 3 \\
\hline Total & 90 \\
\hline
\end{tabular}

Tabla 5 - Frecuencia absoluta de la versión de la publicación de las obras infantiles

Los resultados descritos en esta tabla señalan que: (a) se registran 87 obras infantiles originales; (b) con relación a las traducciones, se identifica un total de tres libros infantiles.

Respecto a la Figura 7, esta investigación confirma una gran incidencia de obras originales en el corpus teoricus de los libros infantiles publicados en Brasil.

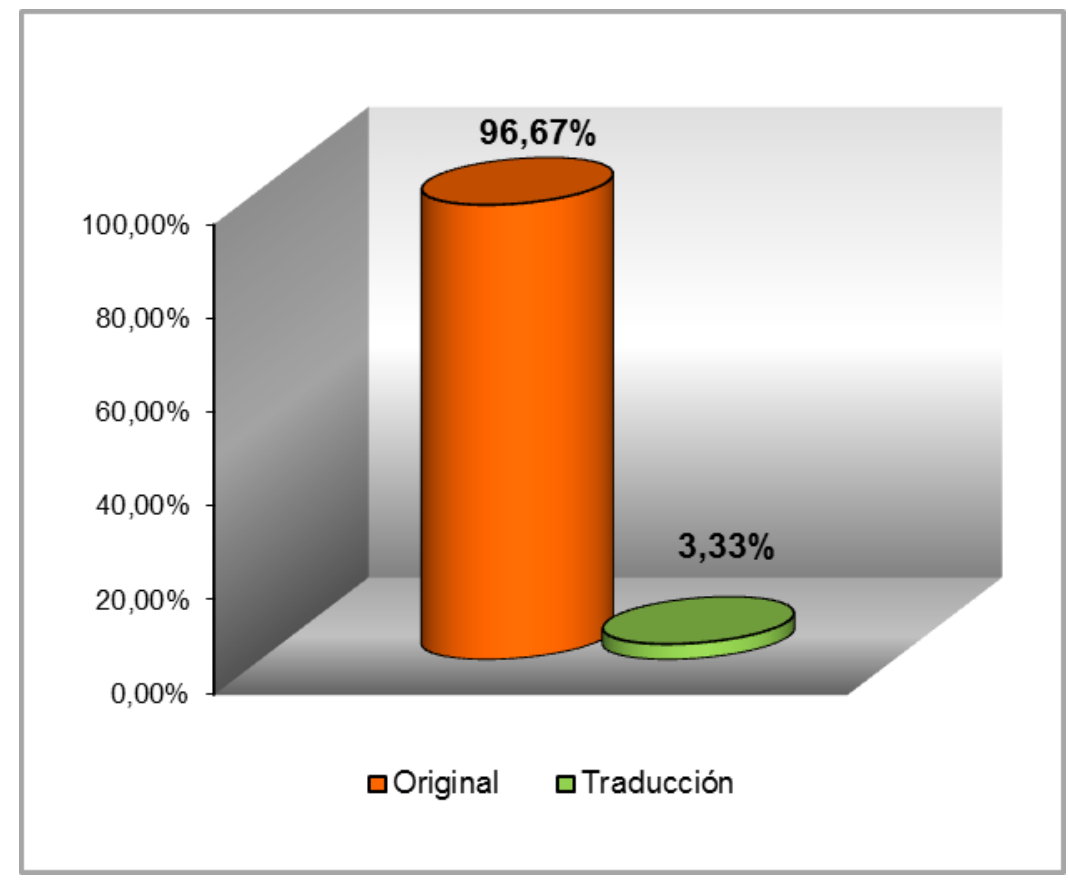

Figura 7 - Representación gráfica de la frecuencia relativa de la versión de la publicación de las obras infantiles 
A partir de los datos descritos en la Figura 7 se puede constatar que la mayoría $(96,67 \%)$ de las obras infantiles publicadas en Brasil es original. En cambio, solamente un 3,33\% de los libros infantiles con temática relacionada con la Educación Física y el Deporte son traducciones. De éstas, se identifica un total de dos idiomas originales, que presentan la incidencia siguiente: inglés, con dos obras infantiles, y, francés, con una obra infantil.

\section{Conclusiones}

A modo de recapitulación, el análisis de las 90 obras infantiles publicadas en Brasil presenta las siguientes conclusiones:

- El género literario predominante en los libros publicados en Brasil es la narrativa.

- El papel es el soporte predominante en los libros infantiles publicados en este país.

- Respecto a la paginación, en Brasil prevalecen los libros no paginados.

- Poco más de la mitad de las obras infantiles está vinculada a una colección sobre deportes.

- La mayoría de las obras infantiles publicadas en Brasil es original.

Por ende, se puede considerar que el conocimiento de las características de los libros infantiles con temática relacionada con la Educación Física y el Deporte es un importante paso para la construcción de una base de datos de literatura infantil en lengua portuguesa dirigida al ámbito de la Educación Física y del Deporte en Brasil.

\section{Agradecimiento}

"El presente artículo fue finalizado con el apoyo del CNPq, Conselho Nacional de Desenvolvimento Científico e Tecnológico - Brasil."

\section{Referencias}

Abril Villalba, M. (2005). Sonido y sentido. La poesía en la construcción lingüística y literaria. En M. Abril Villalba (Coord.), Lectura y literatura infantil y juvenil: Claves (pp. 199-224). Archidona, Málaga: Aljibe.

Bardin, L. (2002). El análisis de contenido (3. ed.). Madrid: Akal.

Botelho, R. G. (2010). Educación Física y literatura infantil: Posibilidades de utilización en el ámbito escolar (2 v.). (Tesis doctoral no publicada). Departamento de Didáctica de la Expresión Musical, Plástica y Corporal, Facultad de Ciencias de la Educación de la Universidad Autónoma de Barcelona, España. (Incluye el CD-ROM Banco de libros infantiles de Educación Física y Deporte en España).

Botelho, R. G., \& Oliveira, C. C. (2011). Estudio sobre el análisis de materia en la literatura infantil relacionada con la Educación Física y el Deporte publicada en España. Biblios: revista de Bibliotecología y Ciencias de la Información, 45, 14-27. doi: 10.5195/biblios.2011.35

Chaumier, J. (1993). Técnicas de documentación y archivo. Barcelona: Oikos-Tau.

Fingon, J. C. (2011). Integrating children's books and literacy into the Physical Education curriculum. Strategies: A journal for Physical and Sport Educators, 24(4), 10-13.

Hernández Sampieri, R., Fernández Collado, C., \& Baptista Lucio, P. (2007). Fundamentos de metodología de la investigación. Madrid: McGraw-Hill/Interamericana de España.

López Yepes, J. (2004). Diccionario enciclopédico de ciencias de la documentación (2 vols.). Madrid: Síntesis.

Weiller, K. H., \& Higgs, C. T. (1989). Female learned helplessness in sport: An analysis of children's literature. Journal of Physical Education, Recreation and Dance, 60(6), 65-67. 


\section{Datos del autor}

\section{Rafael Guimarães Botelho}

Investigador de postdoctorado (2012) - Facultad de Educación de la Universidad de San Pablo (USP), Brasil.

Doctorado en Educación Física y Deporte: didáctica y desarrollo profesional (2010), Máster en Iniciación a la Investigación en Educación Física y Deporte: didáctica y desarrollo profesional (2009) y Diploma de Estudios Avanzados en Didáctica de la Expresión Corporal (2009) - Universidad Autónoma de Barcelona (UAB), España.

Perfeccionamiento en Metodología de la Investigación (2003) y Postgrado en Ética Aplicada y Bioética (2003) Fundación Oswaldo Cruz (FIOCRUZ), Brasil.

Licenciado en Educación Física (2002) y Máster en Educación (2006) - Universidad del Estado de Río de Janeiro (UERJ), Brasil.

Profesor del Instituto Federal de Educación, Ciencia y Tecnología de Río de Janeiro (IFRJ), Brasil.

rafaelgbotelho@ig.com.br

rafael.botelho@ifri.edu.br

Recibido - Received: 2012-11-05

Aceptado - Accepted: 2012-12-30

(cc) $\mathbf{E Y}$ New articles in this journal are licensed under a Creative Commons Attribution 3.0 United States License.

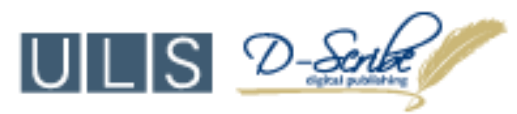

This journal is published by the University Library System of the University of Pittsburgh as part of its D-Scribe Digital Publishing Program and is cosponsored by the University of Pittsburgh Press. 